\title{
Phytochemicals: Current strategies for treating breast cancer (Review)
}

\author{
BRIDG'ETTE B. ISRAEL ${ }^{1 *}$, SYREETA L. TILGHMAN ${ }^{1 *}$, \\ KITANI PARKER-LEMIEUX ${ }^{2}$ and FLORASTINA PAYTON-STEWART ${ }^{3}$
}

\begin{abstract}
${ }^{1}$ Division of Basic Pharmaceutical Sciences, College of Pharmacy and Pharmaceutical Sciences, Florida A\&M University, Tallahassee, FL 32307; ${ }^{2}$ Division of Basic Pharmaceutical Sciences, College of Pharmacy; ${ }^{3}$ Division of Mathematical and Physical Sciences, College of Arts and Sciences, Xavier University of Louisiana, New Orleans, LA 70125, USA
\end{abstract}

Received April 7, 2017; Accepted November 20, 2017

DOI: $10.3892 / \mathrm{ol} .2018 .8304$

\begin{abstract}
Females with early-stage metastatic, estrogen-dependent breast cancer are generally treated with surgery, radiation and chemotherapy, or with more targeted approaches such as aromatase inhibitors (anastrozole or letrozole) or anti-estrogens (tamoxifen). Despite widespread successful usage of these agents for the treatment of breast cancer, resistance, tumor relapse and metastasis remain the principal causes of mortality for patients with breast cancer. While numerous groups have made major contributions toward an improved understanding of resistance mechanisms, the currently insufficient grasp of the most critical pathways involved in resistance is evident in the inability to adequately treat and drastically improve patient outcomes in females with hormone-refractory breast cancer, including triple negative breast cancer. Therefore, further investigation of novel therapeutic approaches is paramount to reveal previously unconsidered agents that could be utilized to treat metastatic disease. Numerous naturally occurring phytochemicals have recently gained interest as potential therapeutic breast cancer agents appear to directly affect estrogen-dependent and estrogen-independent breast cancer cell proliferation, potentially via affecting breast cancer stem cell populations. While numerous natural compounds have exhibited promise, they are limited by their bioavailability. Therefore, to effectively treat future hormone-refractory breast tumors, it is critical to adequately refine and formulate these agents for effective therapeutic use and delivery. Herein, the literature on the current state of phytochemicals is reviewed,
\end{abstract}

Correspondence to: Dr Florastina Payton-Stewart, Division of Mathematical and Physical Sciences, College of Arts and Sciences, Xavier University of Louisiana, 1 Drexel Drive, New Orleans, LA 70125, USA

E-mail: flpayton@xula.edu

*Contributed equally

Key words: phytochemicals, breast cancer, anti-estrogens, aromatase inhibitors including their limitations and potential as targeted therapies for breast cancer.

\section{Contents}

1. Introduction

2. Phytochemicals and breast cancer

3. Phytochemicals and $\operatorname{ER}(+)$ breast cancer

4. Triple negative breast cancer

5. Current treatments for TNBC

6. Phytochemicals used in TNBC treatment

7. Current status and implications of phytochemicals as potential BCSC-targeting therapeutics

8. Pharmaceutical compounding of phytochemicals

9. Conclusions

\section{Introduction}

Breast cancer is the second leading cause of cancer mortality among females, with 246,660 estimated new cases and 40,450 estimated mortalities recorded in the United States in 2016 (1). Today, $1 / 8$ females $(12.5 \%$ ) will develop breast cancer (1). According to the latest World Health Organization report in 2012, by 2030 , the global incidence of breast cancer is projected to increase (2). There are four stages of breast cancer: Cancer in the earliest state is designated stage 0 (carcinoma in situ) and ranges from stage I through IV. Stage IV is the most aggressive stage of the disease. A higher stage implies a more advanced metastatic cancer. Some of the stages are further divided into sub-stages designated A, B and C. When detected early, (i.e., stage I, localized breast cancer), the 5-year survival rate is $100 \%$ (3). It is common for cancer to spread to other organs; breast cancer typically spreads to the lungs, bones, liver or brain (3).

Breast cancer is classified into three main subtypes based on the molecular profiles: i) Hormone receptor-positive [estrogen receptor $(\mathrm{ER})+]$; ii) human epidermal growth factor receptor (EGFR) 2 (HER2)-positive; and iii) triple negative tumors (4). Hormone receptor-positive is a subtype of breast cancer in which the ER is expressed. HER2-positive is a subtype of breast cancer that contains HER2, a member of 
EGFR family. Triple negative breast cancer is a subtype of breast cancer lacking three classic receptors, specifically the ER, progesterone receptor (PR) and HER2. While there are various forms of treatment for breast cancer, treatment is primarily dependent upon the type and stage of the cancer. Current therapeutic strategies include surgery, radiotherapy and adjuvant chemotherapies and hormonal therapies.

Surgery and radiation are used to treat a number of cancers, including breast cancer, to remove, destroy and control the growth of cancer cells in a specific region. Chemotherapy is used as a systematic form of treatment; it is a therapeutic method utilizing drugs to impede the growth of cancer cells. Chemotherapeutic agents are administered orally or by intravenous/intramuscular injection, depending on the type and stage of cancer. Chemotherapy is used to destroy and/or control cancer cells throughout the body. Current treatment methods include: Hormone therapy, such as tamoxifen (Saltamox); aromatase inhibitors, such as anastrozole (Arimidex), exemestane (Aromasin) and letrozole (Femara); and biologic drugs, including ado-trastuzumab emtansine (Kadcyla), lapatinib (Tykerb), palbociclib (Ibrance), pertuzumab (Perjeta) and trastuzumab (Herceptin) (5). Treatment can be administered as a single agent or as a combination, contingent upon the patient's needs. One of the major problems in the treatment of breast cancer is the development of drug resistance, particularly among hormone receptor positive breast cancer.

There are numerous potential reasons for chemotherapy resistance. One reason for resistance is the development of mutated cells from normal cells. Genetic alterations cause aberrant cell activity, thus producing continued mutations and causing cancer cells to arise. Therefore, mutations induce resistance to drugs designed to prevent cancer. Numerous studies have identified a group of genetic mutations responsible for chemotherapy resistance. Cancer cells can hinder drugs from entering affected areas due to these mutations. Studies have determined that resistant cells have high levels of a substance called p-glycoprotein (6). P-glycoprotein is a protein present in cell membranes that functions as a pump to remove toxins from cells. Cells with high p-glycoprotein levels are efficient at cancer drug efflux. Resistance can occur due to insufficient intracellular drug concentrations, which then lead to continued cell proliferation. Cancer cells may also develop mechanisms that allow them to inactivate the anticancer drugs. Furthermore, cancer cells may also acquire novel repair methods for the DNA strand breaks caused by certain anticancer drugs. Recently, numerous studies have identified a subpopulation of cells, termed breast cancer stem cells (CSC), which are accountable for breast cancer reoccurrence and metastasis (7-10). Current research strategies involve identifying novel methods of targeting breast CSCs (11).

\section{Phytochemicals and breast cancer}

There have been advancements in chemotherapeutic agents over the past decade; however, there is still an urgent need to overcome drug resistance. Numerous studies have focused on phytochemicals, which are nonnutritive plant chemicals that have disease-protective or -preventive properties (12-14). These chemicals are non-essential nutrients, meaning they are not required by the human body for sustaining life (15).
Chemicals within these plants have been demonstrated to target molecular subtypes of breast cancer and breast CSCs $(16,17)$. Phytochemicals are non-toxic and have a wide range of biological activity including anti-inflammatory, anti-proliferative, antioxidant and anticancer properties. In this review, we will discuss the current clinical status of phytochemicals and their potential as therapeutics for ER(+) breast cancer, TNBC and breast CSCs. Furthermore, this review addresses the pharmaceutical compounding of phytochemicals and the importance of bioavailability.

\section{Phytochemicals and ER(+) breast cancer}

About $80 \%$ of all breast cancer cases are ER(+). The current method of treatment for ER(+) breast cancer involves hormone therapies such as tamoxifen and aromatase inhibitors (18). Tamoxifen is a selective estrogen receptor modulator (SERM) that prevents cancer by blocking the ER and preventing the binding of hormones. Aromatase inhibitors such as anastrozole, exemestane and letrozole exert their activity through the inhibition of estrogen synthesis. Due to utilizing hormone therapies, the 5-year survival rate for $\mathrm{ER}(+)$ breast cancer is $\sim 10 \%$ higher than ER(-) breast cancer. Conversely, after five years the survival difference decreases and eventually disappears $(19,20)$. Following five years of treatment, female patients with $\mathrm{ER}(+)$ breast cancer develop resistance to hormonal therapies. Thus, numerous studies have focused on the implementation of phytochemicals as adjuvant therapies. There are various phytochemicals that behave as SERMs, including isoflavones, coumestans, lignans and prenylflavonoides. Studies have demonstrated that isoflavones inhibit breast cancer growth, metastasis and induce apoptosis via the modulation of distinct signaling pathways. Daidzein and genistein are isoflavones that inhibit cell proliferation, angiogenesis and induce apoptosis via cell cycle arrest in ER(+) breast cancer cells $(21,22)$. Isoflavones inhibit the ER and EGFR/HER-2 in breast cancer cells. Mai et al (12) revealed that genistein exerts synergistic growth inhibitory effects with tamoxifen and trastuzumab on ER(+) and HER2-overexpressing breast cancer cells (23). Additionally, genistein combined with the synthetic isoflavone ipriflavone inhibits osteolytic bone metastases and improves bone microstructure by suppressing cancer cell growth. The combination of isoflavones and synthetic isoflavones inhibits tumorigenesis $(24,25)$.

Epigallocatechin gallate (EGCG) is a polyphenolic catechin, a phytochemical present in green tea (12). It has been demonstrated to suppress growth and angiogenesis and to increase apoptosis in breast cancer cells (26). EGCG also inhibits migration/invasion and synergizes with tamoxifen, resveratrol and histone deacetylase (HDAC) inhibitors to induce MCF-7 cell death [ER(+) cell line] (27). Flavonols, including quercetin and kaempferol, are phytochemicals present in fruits and vegetables, which possess anti-proliferative activity against breast cancer cells at certain concentrations. Kaempferol inhibits the proliferation of MCF-7 cells at higher doses (50-100 $\mu \mathrm{M})$, whereas it stimulates cell proliferation at lower doses $(5-10 \mu \mathrm{M})$. Yeh et al (28) determined that a combination of quercetin and carboxyamidotriazole has a synergistic effect on cell death in breast cancer. Studies have demonstrated that phytochemicals such as resveratrol, lignans, curcumin and 
carotenoids inhibit the proliferation and increase the apoptosis of breast cancer cells (29-36). Curcumin, a component of turmeric, has been demonstrated to reduce metastasis and interacts with various molecular targets, including $\mathrm{NF} \kappa \mathrm{B}$ and $\mathrm{Wnt} / \beta$-catenin $(34,35)$. A number of phytochemicals are currently in clinical trials for their potential inhibitory effects against breast cancer (36). Future studies are required in order to understand the role of estrogenic phytochemicals and their use as antineoplastic agents for estrogen-dependent vs. hormone-refractory breast cancer.

\section{Triple negative breast cancer}

Triple negative breast cancer (TNBC) is one of the most aggressive forms of breast cancer $(37,38)$. TNBC is derived from epithelial cells and is further characterized by the absence of the ER, PR and the HER2/neu receptor. Although TNBC exhibits some heterogeneity, $72-82.1 \%$ of TNBC tumors reportedly belong to the basal/myoepithelial breast cancer subgroup, which indicates that the epithelial-mesenchymal transition (EMT) has occurred in these cells, and significant genetic alterations have occurred $(39,40)$. TNBC cells acquire a basal-like phenotype as a result of stromal-cancer cell crosstalk, escape death and become more invasive $(41,42)$. Due to the metastatic nature of TNBC cells, there is a requirement for improved targeted therapies for the better management of metastatic breast cancer.

\section{Current treatments for TNBC}

At present, there are no standard guidelines for TNBC treatment. The heterogeneity of TNBC gives rise to various traits that lead to distinct clinical behavior based on the molecular profile of the tumor. Current cytotoxic therapies include taxanes, anthracyclines and platinum agents, particularly cisplatin and carboplatin. Targeted therapies include antiangiogenic agents, tyrosine kinase inhibitors, PARP inhibitors and EGFR inhibitors. Izabepilone and capecitabine combination therapies may also be used. Data indicate that there has been no substantial improvement in patient outcomes. An improved understanding of the tumor microenvironment is critical for the future treatment of cancer and the prevention of recurrence $(43,44)$.

\section{Phytochemicals used in TNBC treatment}

Fresh low bush blueberries (Vaccinium angustifolium) were weighed, juiced and prepared, and expressed as gallic acid equivalents, as described by the Folin-Ciocalteu method (45). The blueberry extract was used to treat TNBC cell lines and a TNBC mouse xenograft model. The results demonstrated that the blueberry extract significantly decreased cell proliferation and tumor volume, and increased apoptosis, as indicated by the evaluation of Ki-67 and caspase- 3 in TNBC cells in vitro and in vivo. The data also indicated a decrease in TNBC cell migration through evaluating modulation of the PI3K/AKT/NFкB pathway (Fig. 1). It was also noted that ingestion of whole blueberries significantly decreased tumor volume in vivo. Additionally, the data indicated that blueberries as daily nutraceutical (6 ounces/day) may be

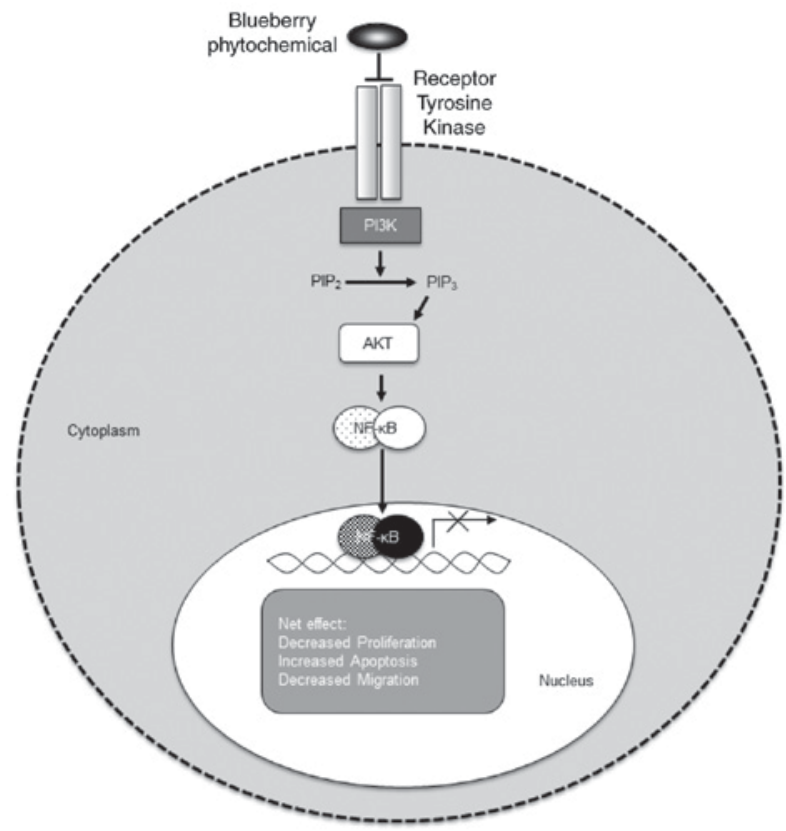

Figure 1. Schematic representation depicting the net effect of blueberry extract on targeting the $\mathrm{PI} 3 \mathrm{~K} / \mathrm{AKT} / \mathrm{NF}-\kappa \mathrm{B}$ pathway in triple negative breast cancer. PI3K, phosphoinositide 3 kinase; AKT, protein kinase $\mathrm{B}$; NF- $\mathrm{NB}$, nuclear factor- $\kappa \mathrm{B}$.

beneficial in cancer prevention (46). The anticancer activity of Mangifera zeylanica (MZ), and of flavonoids from the bark of this plant, endemic to Sri Lanka, was demonstrated in TNBC (47); other prior reports have also indicated the antiproliferative effects of flavonoids in TNBC (48). The bark extracts contain polyphenols and flavonoids, and were used to perform dose-dependent studies to explore the cytotoxic effects of MZ. Caspase- 3 and -7 were activated in TNBC models when the cells were exposed to MZ, leading to apoptosis, which was confirmed by measuring the DNA fragmentation, the expression of pro-apoptotic p53 and Bax, and the expression of anti-apoptotic survivin (47).

Therefore, the evidence suggests that phytochemicals exhibit chemotherapeutic and chemopreventive properties in TNBC (48). Further investigations into the use of phytochemicals as chemotherapeutic agents are warranted.

\section{Current status and implications of phytochemicals as potential BCSC-targeting therapeutics}

While endocrine therapy (i.e., aromatase inhibitors and anti-estrogens) has been demonstrated as an effective first-line therapy for female patients with hormone-dependent breast cancer, it is limited by the development of resistance, and the presence of a rare, sub-population of chemotherapy- and radiation-resistant breast cancer stem cells (BCSC) (49). As BCSCs are able to survive therapy, recurrence may ensue following the treatment of solid tumors due to the self-renewal and multi-lineage differentiation properties of the cells $(50,51)$. It has been reported that standard chemotherapy can increase the percentage of CD $44^{\text {high }} / \mathrm{CD} 24^{\text {low }}$ (BCSC markers) tumor cells in patients with breast cancer (52); thus, alternative therapeutic strategies are critical. The drug-resistant nature of $\mathrm{BCSC}$ has led to increased interest in the utilization of natural 
dietary supplements as alternative and/or adjuvant therapies for existing breast tumors (53). However, the dietary intake of natural agents such as certain phytochemicals, which have been demonstrated to act as estrogens, may be of concern among female patients with $\mathrm{ER}(+)$ breast cancer. Therefore, it is critical to understand the role of phytochemicals as BCSC-targeting agents. Herein is presented a discussion of the current status and implications of phytochemicals as potential BCSC-targeting therapeutics.

The in vitro mammosphere formation assay was originally developed by Dontu et al (54), whereby breast cancer cells are grown in suspension in a low-adhesive environment. The cells aggregate into spheres (i.e., mammospheres or tumorspheres) while suspended to facilitate attachment and differentiation; after long-term culture, mammospheres produce a functional mammary tree structure. This assay is routinely utilized to study mechanistic signaling pathways associated with BCSC and possible therapeutic intervention routes. Rhodiola is a perennial plant that grows in barren soil in the high tundra regions of Siberia and the highlands of Tibet and has been used in traditional eastern medicine (55). There are $\sim 90$ different Rhodiola plant species, all exhibiting a variety of health-promoting effects. Rhodiola exhibit anticancer (54), cardioprotective $(56,57)$ and antidepressant $(58-63)$ properties. Recent studies by Tu et al (64) demonstrated $R$. crenulata induces cell death and inhibits the growth of breast cancer cell lines, as well as reduces the formation and maintenance of MCF-7 (65) and MDA-MB 231 tumor spheres (66). These studies suggested that while components of $R$. crenulata contain estrogens, their use in the treatment of ER(+) breast cancer cells could still be protective.

Previous studies into the phytochemical cajanin stilbene acid (CSA), isolated from pigeon pea (Cajanus cajan) leaves, revealed antioxidant (67) and antimicrobial activity (68), as well as cancer cell cytotoxicity $(69,70)$. Recently, numerous phytochemicals were comparatively analyzed with regard to their effects on BCSC viability. Shikonin, also known as Zicao (purple gromwell), the dried root of Lithospermum erythrorhizon and artesunate (a carbonyl reduced, semi-synthetic derivative of the anti-malarial compound artemisinin isolated from the sweet wormwood plant Artemisia annua) were compared with CSA. Two CSA derivatives were identified to be more effective at inhibiting the viability of MCF-7 cells cultured as tumor spheres, as compared with MCF-7 cells cultured as a monolayer (71). By contrast, $48 \mathrm{~h}$ following Shikonin treatment, the CD $44^{\text {high }} / \mathrm{CD} 24^{\text {low }}$ cells were enriched in the surviving MCF-7 cell population. The results demonstrated that while certain phytochemicals can specifically target the formation and viability of BCSCs, others exhibit initial cytotoxicity towards BCSC and then develop resistance following long-term exposure.

A recent study evaluated the cytotoxic effects of five phytochemicals including 6-gingerol, 6-shogaol, 5-hydroxy3,6,7,8,3',4'-hexamethoxyflavone, nobiletin and pterostilbene on MCF-7 breast cancer cells and BCSCs (72). Results demonstrated 6-gingerol, 6-shogaol and pterostilbene selectively killed the BCSC population while 6-shogaol and pterostilbene induced cell necrosis-associated events such as membrane injury and bleb formation in BCSC, inhibited mammosphere formation and increased the sensitivity of isolated BCSCs to chemotherapeutic drugs. Notably, 6-shogaol and pterostilbene significantly increased the anticancer activity of paclitaxel. The underlying mechanism suggested that decreased stemness could be attributed to decreased CD44 expression, promotion of $\beta$-catenin phosphorylation via the inhibition of hedgehog/Akt/GSK3 $\beta$ signaling, and decreased c-Myc and cyclin D1 expression. This further suggested that 6-shogaol and pterostilbene-activated $\beta$-catenin degradation reduced CD44 activity, thereby affecting the maintenance of BCSCs and further inducing the loss of tumorigenicity.

Due to the inherently resistant nature of BCSCs and their involvement in metastasis, it is increasingly imperative to develop therapies able to directly target these cells. While it is understood that certain soy phytochemicals, including glyceollins, target the EMT (14,73) (Fig. 2), their impact on BCSCs remains unclear. However, phytochemicals possess anticancer properties, and represent a promising approach and novel paradigm for potential BCSC-targeting therapeutics. It is important to develop these agents for use in combination with currently approved endocrine and/or standard chemotherapies with improved bioavailability.

\section{Pharmaceutical compounding of phytochemicals}

Phytochemicals are promising agents for the treatment of estrogen-dependent and -independent breast cancer; however, numerous natural agents have poor bioavailability and, thus, require additional strategies to enhance their efficacy. Understanding of the principles of pharmaceutics is vital for the proper preparation of any drug delivery system or dosage; the pharmaceutical compounding of phytochemicals is no exception. Though the active ingredients are naturally occurring, when administered they exhibit similar pharmacokinetic profiles to chemicals compounded for administration via the same route (74). First pass metabolism is of great concern when a drug or active ingredient is administered orally (75). This phenomenon results in the marked reduction of drug concentration due to hepatic and intestinal drug degradation. Much of the initial concentration is eliminated or removed from the blood before it ever reaches the systemic circulation.

Numerous chemotherapeutic agents are formulated for oral and intravenous administration. Depending on the type of cancer and progression of the disease, patients may be prescribed a single or combination drug regimen (76). Combination therapies typically consist of an oral and intravenous administration of drug entities. For example, methotrexate is given intravenously while cyclophosphamide is orally administered. Conversely, phytochemicals are predominantly administered orally. Phytochemicals are present in high concentrations in fruits and vegetables; as they are consumed as part of a healthy diet, it is logical that compounds formulated to deliver these cancer-fighting agents would utilize the same metabolic routes.

The extent of a drug's solubility in a given pharmaceutical vehicle affects the pharmacokinetics of the active drug moiety. Pharmacokinetics is the study and characterization of the time-course of the absorption, distribution, metabolism and elimination of drugs (77). Solubility and bioavailability are the pharmaceutical compounding parameters of concern. If the compound is not soluble in the transport vehicle, this will affect the bioavailability of the drug or active ingredient(s) (78). Understanding the dynamics of these parameters is crucial when determining the dose regimens and avoiding toxicity. 


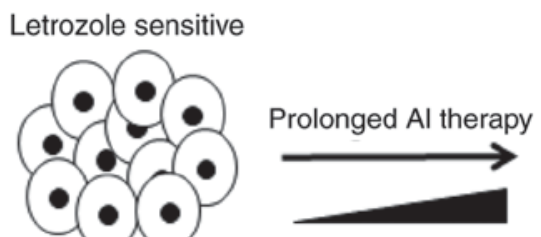

EMT
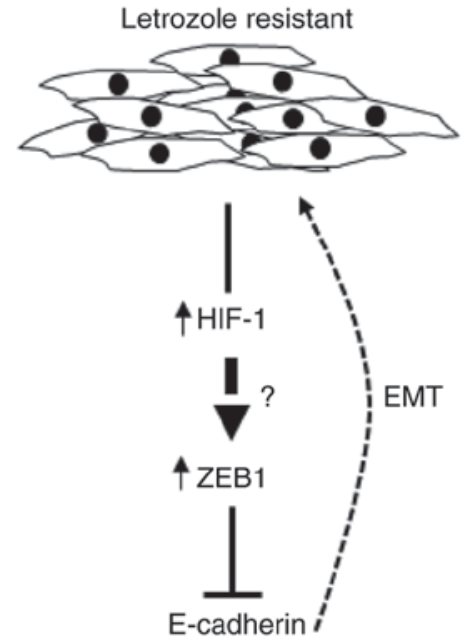
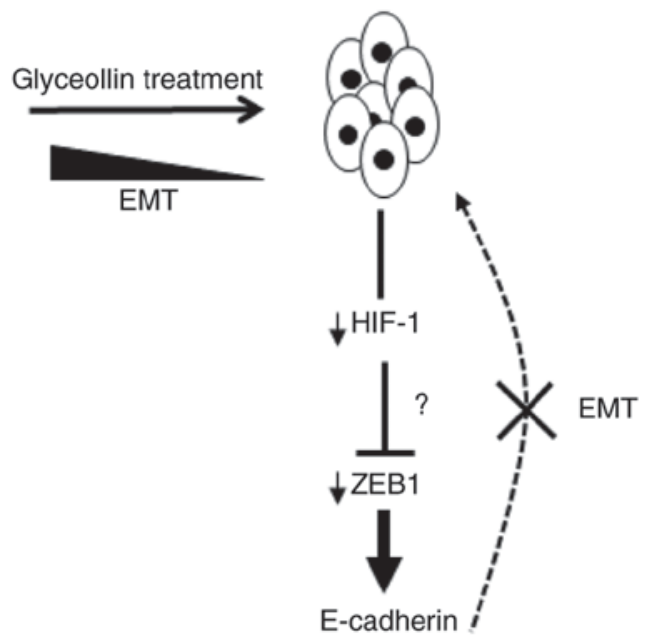

Figure 2. Proposed model of glyceollin-induced reversion of EMT in letrozole-resistant breast cancer cells. As epithelial breast cancer cells that overexpress aromatase are exposed to prolonged AI therapy (i.e., letrozole) they acquire AI resistance, estrogen independence and undergo morphological changes that are associated with EMT. Once resistance occurs, the levels of HIF-1 are increased, which then controls the expression of ZEB1. ZEB1 expression is induced, which in turn suppresses E-cadherin and induces EMT. However, when letrozole-resistant cells are exposed to glyceollin they undergo morphological changes that are associated with an epithelial-like phenotype, accompanied by a decrease in HIF-1 expression levels. This then causes a decrease in ZEB1 expression, allowing the de-suppression of E-cadherin and the inhibition of EMT. EMT, epithelial to mesenchymal transition; AI, aromatase inhibitor; HIF-1, hypoxia-inducible factor-1; ZEB1, zinc finger E-box-binding homeobox 1.

The potential drug release from a specific delivery system or dosage form can be determined via in vitro and in vivo experiments. In vitro testing could include the dissolution data of orally administered dosage forms. The United States Pharmacopeia's protocol for dissolution testing is a standard that can be utilized to customize and design the research procedure (79). In vivo testing should include experiments that provide absorption data, with consistent trends that can be used to further quantify the rate and degree of absorption. Bioavailability is the fraction of drug expected to enter the systemic circulation from a given dosage. This comparative variable is calculated using intravenous administration as a reference. Intravenous administration of a drug allows the total dose amount to enter the blood stream.

Theoretically, this is the ideal scenario for $100 \%$ bioavailability, denoting intravenous delivery the gold standard. The area under the curve (AUC) for the dosage form in question and the intravenous form of the same drug are compared, with the intravenous form as the standard. It is imperative that the pharmacokinetic data are properly identified and interpreted to ensure accurate calculation of the bioavailability factor. The bioavailability factor can be expressed mathematically, as follows:

$$
\mathrm{AUC}_{\text {(given dosage form) }} / \mathrm{AUC}_{\text {(intravenous dosage form) }}
$$

This factor predicts how much of the administered drug will be readily available for absorption. The calculated bioavailability can be multiplied by the recommended dose to yield the quantity of the dose that is available for absorption. For example, if drug X has a bioavailability factor of 0.65 and the administered dose is $100,65 \mathrm{mg}$ of drug $\mathrm{X}$ is expected to enter the systemic circulation.

Dose x Bioavailability Factor=Drug Quantity Entering Systemic Circulation, $100 \mathrm{mg}$ x $0.65=65 \mathrm{mg}$.
The intravenous form of the drug is the reference; therefore, the bioavailability factor for the intravenous administration of any drug is 1 .

Numerous groups have reported low bioavailability from orally administered phytochemicals. Manach et al (80) identified that low bioavailability could be attributed to multiple parameters. Liver and gut metabolism, urinary excretion and the patient's genetic profile are among the factors that account for a decreased quantity of phytochemicals reaching the systemic circulation. If these compounds are unable to enter systemic circulation, the anticipated therapeutic response will not occur.

In vitro and in vivo studies demonstrate that phytochemicals can increase the apoptosis of cancer cells and reduce metastasis (81-84). Phytochemicals will not benefit the patient if they are not compounded in a manner that protects them from degradation. Therefore, consideration has been given to formulating phytochemicals in order to increase bioavailability and clinical efficacy. Developing mutual prodrugs, orally co-delivering phytochemicals, and administering phytochemicals as a topical or parental formulation are all attempts to provide alternatives to the current treatment options (85-87).

A mutual prodrug may provide a more promising orally administered phytochemical. Siddiqui and Mukhtar (88) suggested combining trastuzumab with a potent polyphenolic catechin as a plausible novel strategy for treating patients with HER2-overexpressing breast cancer. Formulating phytochemicals into nanoparticles has proven effective at increasing bioavailability and represents a viable targeted therapy option. Manzoor et al (89) reported a significant increase in the bioavailability of doxorubicin when thermally sensitive liposomes were released into a tumor. Research findings and advances are providing methodologies for the development of novel and individualized phytochemical-based treatments for patients with cancer. 
Combining cancer therapies is a well-documented practice; this premise is considered a plausible means to optimize the outcomes from phytochemical treatment. Improved therapeutic response, reduction in dose, and toxicity are expected due to multiple mechanisms being targeted (90). The ideal combination will contain active components working on different sites, which should reduce the possibility of acquired tolerance to the therapy or postpone the time before resistance is detected.

Gundala et al (91) compared the traditional (oral) route of administering phytochemicals to the topical and parental routes. The findings from this study suggest there is an increase in dose precision and clinical efficacy when phytochemicals are administered via the parental or topical route. Parental delivery markedly increases bioavailability while topical delivery boosts local activity. These attributes primarily exist as transport via either route of administration minimizes the first pass metabolism effect (92).

The various routes of administration (oral, topical, and parental) utilized to transport active ingredients and drug entities are well established. Combining the routes and dosage forms (capsules, creams, and liposomes) presents a number of options for investigation, all of which exhibit promise for the delivery of phytochemicals and address the requirement to increase bioavailability.

\section{Conclusions}

Despite advances in the treatment of breast cancer, there remains a requirement to overcome therapeutic resistance and develop novel treatments for metastatic breast cancer. Recent studies have demonstrated that BCSCs may be responsible for resistance. Crosstalk between the primary tumor and the stroma or microenvironment is reportedly potentially responsible for the migration and invasive nature of metastatic breast cancer. In order to address these concerns, attention must be focused on compounds that specifically affect various molecular targets associated with stem cells and the metastatic tumor microenvironment. Phytochemicals have proven effective at targeting numerous signaling pathways and BCSCs in breast cancer. Utilizing well-formulated phytochemicals in nanoparticles, in combination with currently approved endocrine and/or standard chemotherapies, may prove a feasible method of overcoming resistance and targeting BCSCs in metastatic breast cancer. Therefore, future studies should involve varied drug delivery methods, including nanoparticles and liposomes, with phytochemicals in combination with current methods of treatment.

\section{Acknowledgements}

The authors would like to thank Dr Leyte Winfield (Spelman University) and Professor Guangdi Wang (Xavier University of Louisiana) for discussion and communications. We thank the Xavier Faculty Writing Group (XULA-WG) for inspiring us to see this work to completion.

\section{Funding}

This study was supported through funds providing researchers with release time in part by the NIH (grant no. 1SC2GM099599-01A1; SLT) and the Florida A\&M NIH
RCMI grant G12MD007582; BBI and SLT). Funding was also received from the Louisiana Cancer Research Consortium and the NIH-RCMI (grant no. 8G12MD007595-04) from the National Institute on Minority Health and Health Disparities and (grant nos. U54MD008149 and G12MD007582, sub-award 13-14-MB-G007RN0A-XU-KPL; KPL), the Bureau of Health Professions, Health Resources and Services Administration, Department of Health and Human Services (grant no. D34HP00006; IBB), the National Science Foundation (grant no. DBI-0829236; IBB), the NIGMS-BUILD (grant no. 8UL1GM118967; FPS) and the RCMI (grant no. 5G12MD007595; FPS) from the National Institute on Minority Health and Health Disparities and the Louisiana Cancer Research Consortium. The contents are solely the responsibility of the authors and do not necessarily represent the official views of the Louisiana Cancer Research Consortium or the NIH.

\section{Availability of data and materials}

Not applicable.

\section{Authors' contributions}

BBI researched and summarized the data regarding pharmaceutical compounding of phytochemicals. SLT researched and summarized current status and implications of phytochemicals as potential BCSC-targeting therapeutics. KPL researched and summarized current treatments for TNBC and phytochemicals used in TNBC. FPS researched and summarized phytochemicals and breast cancer and ER(+) breast cancer.

\section{Ethics approval and consent to participate}

Not applicable.

\section{Consent for publication}

Not applicable.

\section{Competing interests}

The authors declare that they have no competing interests.

\section{References}

1. Siegel RL, Miller KD and Jemal A: Cancer statistics, 2016. CA Cancer J Clin 66: 7-30, 2016.

2. Bray F, Jemal A, Grey N, Ferlay J and Forman D: Global cancer transitions according to the human development index (2008-2030): A population-based study. Lancet Oncol 13: 790-801, 2012.

3. DeSantis C, Siegel R and Jemal A: Cancer treatment and survivorship facts and figures 201s.-2014-2015. American Cancer Society, Atlanta, GA, 2014. https://www.cancer.org/content/ dam/cancer-org/research/cancer-facts-and-statistics/ cancer-treatment-and-survivorship-facts-and-figures/cancertreatment-and-survivorship-facts-and-figures-2014-2015.pdf.

4. Baselga J and Swain SM: Novel anticancer targets: Revisiting ERBB2 and discovering ERBB3. Nat Rev Cancer 9: 463-475, 2009.

5. Carels N, Spinassé LB, Tilli TM and Tuszynski JA: Toward precision medicine of breast cancer. Theor Biol Med Model 13: 7, 2016.

6. Katayama K, Noguchi K and Sugimoto Y: Regulations of P-Glycoprotein/ABCB1/MDR1 in human cancer cells. New J Sci 2014: 1-10, 2014. 
7. Chen K, Huang YH and Chen JL: Understanding and targeting cancer stem cells: Therapeutic implications and challenges. Acta Pharmacol Sin 34: 732-740, 2013.

8. Nie S, McDermott SP, Deol Y, Tan Z, Wicha MS and Lubman DM: A quantitative proteomics analysis of MCF7 breast cancer stem and progenitor cell populations. Proteomics 15: 3772-3783, 2015.

9. Velasco-Velázquez MA, Popov VM, Lisanti MP and Pestell RG: The role of breast cancer stem cells in metastasis and therapeutic implications. Am J Pathol 179: 2-11, 2011.

10. Li W, Ma H, Zhang J, Zhu L, Wang C and Yang Y: Unraveling the roles of CD44/CD24 and ALDH1 as cancer stem cell markers in tumorigenesis and metastasis. Sci Rep 7: 13856, 2017.

11. Liu S and Wicha MS: Targeting breast cancer stem cells. J Clin Oncol 28: 4006-4012, 2010.

12. Mai Z, Blackburn GL and Zhou JR: Genistein sensitizes inhibitory effect of tamoxifen on the growth of estrogen receptor-positive and HER2-overexpressing human breast cancer cells. Mol Carcinog 46: 534-542, 2007.

13. Limer JL, Parkes AT and Speirs V: Differential response to phytoestrogens in endocrine sensitive and resistant breast cancer cells in vitro. Int J Cancer 119: 515-521, 2006.

14. Carriere PP, Llopis SD, Naiki AC, Nguyen G, Phan T, Nguyen MM, Preyan LC, Yearby L, Pratt J, Burks H, et al: Glyceollin I reverses epithelial to mesenchymal transition in letrozole resistant breast cancer through ZEB1. Int J Environ Res Public Health 13: ijerph13010010, 2015.

15. Liu RH: Health benefits of fruit and vegetables are from additive and synergistic combinations of phytochemicals. Am J Clin Nutr 78 (3 Suppl): 517S-520S, 2003

16. Dandawate P, Padhye S, Ahmad A and Sarkar FH: Novel strategies targeting cancer stem cells through phytochemicals and their analogs. Drug Deliv Transl Res 3: 165-182, 2013.

17. Siddiqui JA, Singh A, Chagtoo M, Singh N, Godbole MM and Chakravarti B: Phytochemicals for breast cancer therapy: Current status and future implications. Current Cancer Drug Targets 15: 116-135, 2015

18. Toss A and Crostofanilli M: Molecular characterization and targeted therapeutic approaches in breast cancer. Breast Cancer Res 17: 60, 2015

19. Fisher B, Redmond C, Fisher ER and Caplan R: Relative worth of estrogen or progesterone receptor and pathologic characteristics of differentiation as indicators of prognosis in node-negative breast cancer patients: Findings from national surgical adjuvant breast and bowel project protocol B-06. J Clin Oncol 6: 1076-1087, 1988

20. Bentzon N, Düring M, Rasmussen BB, Mouridsen H and Kroman N: Prognostic effect of estrogen receptor status across age in primary breast cancer. Int J Cancer 122: 1089-1094, 2008

21. Shao ZM, Wu J, Shen ZZ and Barsky SH: Genistein exerts multiple suppressive effects on human breast carcinoma cells Cancer Res 58: 4851-4857, 1998.

22. Li Y, Upadhyay S, Bhuiyan M and Sarkar FH: Induction of apoptosis in breast cancer cells MDA-MB-231 by genistein. Oncogene 18: 3166-3172, 1999.

23. Choi EJ and Kim GH: Antiproliferative activity of daidzein and genistein may be related to ER $\alpha / \mathrm{c}$-erbB-2 expression in human breast cancer cells. Mol Med Rep 7: 781-784, 2013.

24. Lattrich C, Lubig J, Springwald A, Goerse R, Ortmann O and Treeck O: Additive effects of trastuzumab and genistein on human breast cancer cells. Anticancer Drugs 22: 253-261, 2011.

25. Hooshmand S, Khalil DA, Murillo G, Singletary K, Kamath SK and Arjmandi BH: The combination of genistin and ipriflavone prevents mammary tumorigenesis and modulates lipid profile. Clin Nutr 27: 643-648, 2008

26. Gu JW, Makey KL, Tucker KB, Chinchar E, Mao X, Pei I, Thomas EY and Miele L: ECGC, a major green tea catechin suppresses breast tumor angiogenesis and growth via inhibiting the activation of HIV-1 $\alpha$ and NFKB, and VEGF expression. Vasc Cell 5: 9, 2013

27. Sen T and Chatterjee A: Epigallocatechin-3-gallate (EGCG) downregulates EGF-induced MMP-9 in breast cancer cells: Involvement of integrin receptor $\alpha 5 \beta 1$ in the process. Eur J Nutr 50: 465-478, 2011

28. Yeh YA, Herenyiova M and Weber G: Quercetin: Synergistic action with carboxyamidotriazole in human breast carcinoma cells. Life Sci 57: 1285-1292, 1995.

29. Papoutsi Z, Kassi E, Tsiapara A, Fokialakis N, Chrousos GP and Moutsatsou P: Evaluation of estrogenic/antiestrogenic activity of ellagic acid via the estrogen receptor subtypes ERalpha and ERbeta. J Agric Food Chem 53: 7715-7720, 2005.

30. Warburg O: On the origin of cancer cells. Science 123: 309-314, 1956
31. Amon LM, Pitteri SJ, Li CI, McIntosh M, Ladd JJ, Disis M, Porter P, Wong $\mathrm{CH}$, Zhang Q, Lampe $\mathrm{P}$, et al: Concordant release of glycolysis proteins into the plasma preceding a diagnosis of ER+ breast cancer. Cancer Res 72: 1935-1942, 2012.

32. Gomez LS, Zancan P, Marcondes MC, Ramos-Santos L, Meyer-Fernandes JR, Sola-Penna M and Da Silva D: Resveratrol decreases breast cancer cell viability and glucose metabolism by inhibiting 6-phosphofructo-1-kinase. Biochimie 95: 1336-1343, 2013.

33. Cheng AL, Hsu CH, Lin JK, Hsu MM, Ho YF, Shen TS, Ko JY, Lin JT, Lin BR, Ming-Shiang W, et al: Phase I clinical trial of curcumin, a chemopreventive agent, in patients with high-risk or pre-malignant lesions. Anticancer Res 21: 2895-2900, 2001.

34. Taipale $\mathrm{J}$ and Beachy PA: The Hedgehog and Wnt signaling pathways in cancer. Nature 411: 349-354, 2001.

35. Hammerschmidt M, Brook A and McMahon AP: The world according to hedgehog. Trends Genet 13: 14-21, 1997.

36. Sharma RA, Euden SA, Platton SL, Cooke DN, Shafayat A, Hewitt HR, Marczylo TH, Morgan B, Hemingway D, Plummer SM, et al: Phase I clinical trial of oral curcumin: Biomarkers of systemic activity and compliance. Clin Cancer Res 10: 6847-6854, 2004.

37. Toss A and Cristofanilli M: Molecular characterization and targeted therapeutic approaches in breast cancer. Breast Cancer Res 17: 60, 2015.

38. Vella LJ: The emerging role of exosomes in epithelial-mesenchymal-transition in cancer. Front Oncol 4: 361, 2014.

39. Mbeunkui $F$ and Johann DJ Jr: Cancer and the tumor microenvironment: A review of an essential relationship. Cancer Chemother Pharmacol 63: 571-582, 2009

40. Suetsugu A, Honma K, Saji S, Moriwaki H, Ochiya T and Hoffman RM: Imaging exosome transfer from breast cancer cells to stroma at metastatic sites in orthotopic nude-mouse models. Adv Drug Deliv Rev 65: 383-390, 2013

41. Jiang Z, Jones R, Liu JC, Deng T, Robinson T, Chung PE, Wang S, Herschkowitz JI, Egan SE, Perou CM and Zacksenhaus E: RB1 and $\mathrm{p} 53$ at the crossroad of EMT and triple-negative breast cancer. Cell Cycle 10: 1563-1570, 2011.

42. Ge R, Tan E, Sharghi-Namini S and Asada HH: Exosomes in cancer microenvironment and beyond: Have we overlooked these extracellular messengers? Cancer Microenviron 5: 323-332, 2012.

43. Greening DW, Gopal SK, Mathias RA, Liu L, Sheng J, Zhu HJ and Simpson RJ: Emerging roles of exosomes during epithelial-mesenchymal transition and cancer progression. Semin Cell Dev Biol 40: 60-71, 2015.

44. Yadav BS, Sharma SC, Chanana P and Jhamb S: Systemic treatment strategies for triple-negative breast cancer. World J Clin Oncol 5: 125-133, 2014.

45. Sharma M, Li L, Celver J, Killian C, Kovoor A and Seeram NP: Effects of fruit ellagitannin extracts, ellagic acid, and their colonic metabolite, urolithin A, on Wnt signaling. J Agric Food Chem 58: 3965-3969, 2010.

46. Adams LS, Phung S, Yee N, Seeram NP, Li L and Chen S: Blueberry phytochemicals inhibit growth and metastatic potential of MDA-MB-231 breast cancer cells through modulation of the phosphatidylinositol 3-kinase pathway. Cancer Res 70: 3594-3605, 2010

47. Ediriweer MK, Tennekoon KH, Samarakoon SR, Thabrew I and Dilip De Silva E: A study of the potential anticancer activity of Mangifera zeylanica bark: Evaluation of cytotoxic and apoptotic effects of the hexane extract and bioassay-guided fractionation to identify phytochemical constituents. Oncol Lett 11: 1335-1344, 2016.

48. Johnson KP, Yearby LA, Stoute D, Burow ME, Rhodes LV, Gray M, Carriere P, Tilghman SL, McLachlan JA and Ochieng J: In vitro and in vivo evaluation of novel anticancer agents in triple negative breast cancer models. J Health Care Poor Underserved 24 (1 Suppl): S104-S111, 2013.

49. Kakarala M and Wicha MS: Implications of the cancer stem-cell hypothesis for breast cancer prevention and therapy. J Clin Oncol 26 2813-2820, 2008

50. Zhou BB, Zhang H, Damelin M, Geles KG, Grindley JC and Dirks PB: Tumour-initiating cells: Challenges and opportunities for anticancer drug discovery. Nat Rev Drug Discov 8: 806-823, 2009.

51. Sengupta A and Cancelas JA: Cancer stem cells: A stride towards cancer cure? J Cell Physiol 225: 7-14, 2010.

52. Li X, Lewis MT, Huang J, Gutierrez C, Osborne CK, Wu MF, Hilsenbeck SG, Pavlick A, Zhang X, Chamness GC, et al: Intrinsic resistance of tumorigenic breast cancer cells to chemotherapy. J Natl Cancer Inst 100: 672-679, 2008 
53. Oh J, Hlatky L, Jeong YS and Kim D: Therapeutic effectiveness of anticancer phytochemicals on cancer stem cells. Toxins (Basel) 8: pii: E199, 2016.

54. Dontu G, Abdallah WM, Foley JM, Jackson KW, Clarke MF, Kawamura MJ and Wicha MS: In vitro propagation and transcriptional profiling of human mammary stem/progenitor cells Genes Dev 17: 1253-1270, 2003.

55. Ming DS, Hillhouse BJ, Guns ES, Eberding A, Xie S, Vimalanathan S and Towers GH: Bioactive compounds from Rhodiola rosea (Crassulaceae). Phytother Res 19: 740-743, 2005.

56. Zubeldia JM, Nabi HA, Jiménez del Río M and Genovese J: Exploring new applications for Rhodiola rosea: Can we improve the quality of life of patients with short-term hypothyroidism induced by hormone withdrawal? J Med Food 13: 1287-1292, 2010

57. Wu T, Zhou H, Jin Z, Bi S, Yang X, Yi D and Liu W: Cardioprotection of salidroside from ischemia/reperfusion injury by increasing $\mathrm{N}$-acetylglucosamine linkage to cellular proteins Eur J Pharmacol 613: 93-99, 2009.

58. Amsterdam JD and Panossian AG: Rhodiola rosea L. as a putative botanical antidepressant. Phytomedicine 23: 770-783, 2016.

59. Mao JJ, Li QS, Soeller I, Xie SX and Amsterdam JD: Rhodiola rosea therapy for major depressive disorder: A study protocol for a randomized, double-blind, placebo-controlled trial. J Clin Trials 4: 170, 2014

60. Mao JJ, Xie SX, Zee J, Soeller I, Li QS, Rockwell K and Amsterdam JD: Rhodiola rosea versus sertraline for major depressive disorder: A randomized placebo-controlled trial Phytomedicine 22: 394-399, 2015

61. Muszyńska B, Łojewski M, Rojowski J, Opoka W and Sułkowska-Ziaja K: Natural products of relevance in the prevention and supportive treatment of depression. Psychiatr Pol 49: 435-453, 2015

62. Yang SJ, Yu HY, Kang DY, Ma ZQ, Qu R, Fu Q and Ma SP. Antidepressant-like effects of salidroside on olfactory bulbectomy-induced pro-inflammatory cytokine production and hyperactivity of HPA axis in rats. Pharmacol Biochem Behav 124: 451-457, 2014.

63. Zhang X, Du Q, Liu C, Yang Y, Wang J, Duan S and Duan J: Rhodioloside ameliorates depressive behavior via up-regulation of monoaminergic system activity and anti-inflammatory effect in olfactory bulbectomized rats. Int Immunopharmacol 36: 300-304, 2016.

64. Tu Y, Roberts L, Shetty K and Schneider SS: Rhodiola crenulata induces death and inhibits growth of breast cancer cell lines. J Med Food 11: 413-423, 2008.

65. Bassa LM, Jacobs C, Gregory K, Henchey E, Ser-Dolansky J and Schneider SS: Rhodiola crenulata induces an early estrogenic response and reduces proliferation and tumorsphere formation over time in MCF7 breast cancer cells. Phytomedicine 23: 87-94, 2016.

66. Gauger KJ, Rodriguez-Cortes A, Hartwich M and Schneider SS Rhodiola crenulata inhibits the tumorigenic properties of invasive mammary epithelial cells with stem cell characteristics. J Med Plant Res 4: 446-454, 2010.

67. Wu N, Kong Y, Fu Y,Zu Y, Yang Z, Yang M, Peng X and Efferth T: In vitro antioxidant properties, DNA damage protective activity, and xanthine oxidase inhibitory effect of cajaninstilbene acid, a stilbene compound derived from pigeon pea [Cajanus cajan (L.) Millsp.] leaves. J Agric Food Chem 59: 437-443, 2011.

68. Zu YG, Liu XL, Fu YJ, Wu N, Kong Y and Wink M: Chemical composition of the SFE-CO extracts from Cajanus cajan (L.) Huth and their antimicrobial activity in vitro and in vivo. Phytomedicine 17: 1095-1101, 2010.

69. Fu Y, Kadioglu O, Wiench B, Wei Z, Gao C, Luo M, Gu C, Zu Y and Efferth T: Cell cycle arrest and induction of apoptosis by cajanin stilbene acid from Cajanus cajan in breast cancer cells. Phytomedicine 22: 462-468, 2015.

70. Fu Y, Kadioglu O, Wiench B, Wei Z, Wang W, Luo M, Yang X, $\mathrm{Gu} \mathrm{C}, \mathrm{Zu} \mathrm{Y}$ and Efferth T: Activity of the antiestrogenic cajanin stilbene acid towards breast cancer. J Nutr Biochem 26 : 1273-1282, 2015

71. Seo EJ, Wiench B, Hamm R, Paulsen M, Zu Y, Fu Y and Efferth T: Cytotoxicity of natural products and derivatives toward MCF-7 cell monolayers and cancer stem-like mammospheres. Phytomedicine 22: 438-443, 2015.

72. Wu CH, Hong BH, Ho CT and Yen GC: Targeting cancer stem cells in breast cancer: Potential anticancer properties of 6-shogaol and pterostilbene. J Agric Food Chem 63: 2432-2441, 2015.
73. Lee SH, Jee JG, Bae JS, Liu KH and Lee YM: A group of nove HIF- $1 \alpha$ inhibitors, glyceollins, blocks HIF- $1 \alpha$ synthesis and decreases its stability via inhibition of the PI3K/AKT/mTOR pathway and hsp90 binding. J Cell Physiol 230: 853-862, 2015.

74. Aqil F, Munagala R, Jeyabalan J and Vadhanam MV: Bioavailability of phytochemicals and its enhancement by drug delivery systems. Cancer Lett 334: 133-141, 2013.

75. Aungst BJ: Novel formulation strategies for improving oral bioavailability of drugs with poor membrane permeation or presystemic metabolism. J Pharm Sci 82: 979-987, 1993.

76. Ansel HC and Stoklosa MJ: Pharmaceutical calculations. 12th edition. Lippincott Williams \& WIlkins, Baltimore, MD, pp321-324, 2006.

77. Subramanian AP, Jaganathan SK, Manikandan A, Pandiaraj KN, Gomathi $\mathrm{N}$ and Supriyanto E: Recent trends in nano-based drug delivery systems for efficient delivery of phytochemicals in chemotherapy. RSC Adv 6: 48294-48314, 2016.

78. Vasconcelos T, Sarmento B and Costa P: Solid dispersions as strategy to improve oral bioavailability of poor water-soluble drugs. Drug Discov Today 12: 1068-1075, 2007

79. DeStefano AJ, Hauck WW, Stippler ES, Brown WE, Li C, Huang GG, Jones BJ, O'Hool K, Koch WF and Williams RL: Establishing new acceptance limits for dissolution performance verification of USPC apparatus 1 and 2 using USPC prednisone tablets reference standard. Pharm Res 28: 505-516, 2011.

80. Manach C, Scalbert A, Morand C, Rémésy C and Jiménez L: Polyphenols: Food sources and bioavailability. Am J Clin Nutr 79: 727-747, 2004.

81. Fantini M, Benvenuto M, Masuelli L, Frajese GV, Tresoldi I, Modesti A and Bei R: In vitro and in vivo antitumoral effects of combinations of polyphenols, or polyphenols and anticancer drugs: Perspectives on cancer treatment. Int J Mol Sci 16: 9236-9282, 2015.

82. Wang H, Khor TO, Shu L, Su ZY, Fuentes F, Lee JH and Kong AN: Plants vs. cancer: A review on natural phytochemicals in preventing and treating cancer and their druggability. Anticancer Agents Med Chem 12: 1281-1305, 2012.

83. Hosseini A and Ghorbani A: Cancer therapy with phytochemicals: Evidence from clinical studies. Avicenna J Phytomed 5: 84-97, 2015

84. Ham SL, Nasrollahi S, Shah KN, Soltisz A, Paruchuri S, Yun YH, Luker GD, Bishayee A and Tavana H: Phytochemicals potently inhibit migration of metastatic breast cancer cells. Integr Biol (Camb) 7: 892-800, 2015

85. Mishra B, Patel BB and Tiwari S: Colloidal nanocarriers: A review on formulation technology, types and applications toward targeted drug delivery. Nanomedicine 6: 9-24, 2010.

86. Boonme P, Kaewbanjong J, Amnuaikit T, Andreani T, Silva AM and Souto EB: Microemulsion and microemulsion-based gels for topical antifungal therapy with phytochemicals. Curr Pharm Des 22: 4257-4263, 2016.

87. Kang L, Gao Z, Huang W, Jin M and Wang Q: Nanocarrier-mediated co-delivery of chemotherapeutic drugs and gene agents for cancer treatment. Acta Pharmaceutica Sinica B 5: 169-175, 2015.

88. Siddiqui IA and Mukhtar H: Nanochemoprevention by bioactive food components: A perspective. Pharm Res 27: 1054-1060, 2010.

89. Manzoor AA, Lindner LH, Landon CD, Park JY, Simnick AJ, Dreher MR, Das S, Hanna G, Park W, Chilkoti A, et al: Overcoming limitations in nanoparticle drug delivery: Triggered, intravascular release to improve drug penetration into tumors. Cancer Res 72: 5566-5575, 2012

90. Tsouris V, Joo MK, Kim SH, Kwon IC and Won YY: Nano carriers that enable co-delivery of chemotherapy and RNAi agents for treatment of drug-resistant cancers. Biotechnol Adv 32: 1037-1050, 2014.

91. Gundala SR, Mukkavilli R, Yang C, Yadav P, Tandon V, Vangala S, Prakash S and Aneja R: Enterohepatic recirculation of bioactive ginger phytochemicals is associated with enhanced tumor growth-inhibitory activity of ginger extract. Carcinogenesis 35: 1320-1329, 2014.

92. Shi J, Wang Y and Luo G: Ligustrazine phosphate ethosomes for treatment of Alzheimer's disease, in vitro and in animal model studies. AAPS PharmSciTech 13: 485-492, 2012. 